\title{
Transition in relaxation paths in allosteric molecules: Enzymatic kinetically constrained model
}

\author{
Tetsuhiro S. Hatakeyama $\odot^{*}$ and Kunihiko Kaneko \\ Department of Basic Science, University of Tokyo, 3-8-1 Komaba, Meguro-ku, Tokyo 153-8902, Japan
}

(Received 28 May 2019; published 7 January 2020)

\begin{abstract}
A hierarchy of timescales is ubiquitous in biological systems, where enzymatic reactions play an important role because they can hasten the relaxation to equilibrium. We introduced a statistical physics model of interacting spins that also incorporates enzymatic reactions to extend the classic model for allosteric regulation. Through Monte Carlo simulations, we found that the relaxation dynamics are much slower than the elementary reactions and are logarithmic in time with several plateaus, as is commonly observed for glasses. This is because of the kinetic constraints from the cooperativity via the competition for an enzyme, which has a different affinity for molecules with different structures. Our model showed symmetry breaking in the relaxation trajectories that led to inherently kinetic transitions without any correspondence to the equilibrium state. In this Rapid Communication, we discuss the relevance of these results for diverse responses in biology.
\end{abstract}

DOI: 10.1103/PhysRevResearch.2.012005

\section{INTRODUCTION}

Biological systems are known to have a hierarchy of timescales [1]. Ordinarily, the timescale of biochemical reactions is of the subsecond order, that of organisms' behaviors is of the order of seconds to hours, and that of lifespans is of the order of years. How organisms fill the gaps between such timescales remains one of the most important problems in biophysics.

As long as the Michaelis-Menten kinetics is satisfied and the concentration of a substrate is saturated, which is ordinary in cells, the gap of timescales between biochemical reactions and organisms' behaviors is hardly filled. Recent studies, however, reported that the kinetics of the multisite modification of proteins does not always follow ordinary MichaelisMenten kinetics [2-6]. For example, in the Erk/Elk-1 signaling pathway, the timescales of the phosphorylation reactions are broadly distributed among multiple sites, and the phosphorylation speed of each site depends on both the site itself and the modification of other sites [2]. It is shown theoretically that multisite modification and the competition for limited enzyme abundances can change the kinetics as well as the steady-state modification level [3-5]. Notably, sequential multisite modification has been reported to generate a variety of timescales, some of which are much slower than the enzymatic turnover rate [5].

Such regulation of the modification kinetics, including the slow dynamics, is considered to result from intermolecular cooperativity. Although a dynamical-system model with chemical kinetics has previously been proposed for the average relaxation process, a model and analysis that go beyond

\footnotetext{
*hatakeyama@ complex.c.u-tokyo.ac.jp

Published by the American Physical Society under the terms of the Creative Commons Attribution 4.0 International license. Further distribution of this work must maintain attribution to the author(s) and the published article's title, journal citation, and DOI.
}

dynamical systems are required to reveal the intermolecular cooperativity and the fluctuations in the slow relaxation process.

Concepts from statistical physics may be useful for investigating the slow biochemical dynamics and its fluctuation. Such slow dynamics have been extensively and intensively studied with regard to the physics of glasses [7]. In kinetically constrained models (KCMs), relaxation to the equilibrium is kinetically suppressed without resorting to thermodynamic metastable states [8]. A promising mechanism for kinetically slowed-down processes, then, is control of the enzyme abundance. Because the reaction rate is controlled enzymatically, the lack of an enzyme may suppress the corresponding reactions. Despite the possible relevance of the kinetic constraint concept to biochemical processes, it has not been fully explored due to the lack of a KCM for biological systems.

In this Rapid Communication, to uncover a relationship between the slow dynamics in biology and the kinetic constraint, we introduce an enzymatic kinetically constrained model (eKCM) by adopting the Monod-Wyman-Changeux (MWC) model for multiple modifications of the protein state (Fig. 1), which is the classic established model for concerted allosteric regulation [9] (see also Refs. [10-12] for the origin of allostery). The essence of allostery is represented by a coupling between modification and structure of a molecule. Here, we consider two types allosteric effect: (i) the energetic effect and (ii) the enzymatic (kinetic) effect; the former has been considered in statistical-physics models [13] and the latter has not been considered and is required for eKCM. We demonstrate that even if both effects accelerate reactions, the eKCM counterintuitively showed a slow relaxation to the equilibrium state. A typical time course for relaxation showed multiple plateaus, where the modification progress was transiently frozen far from equilibrium.

\section{MODEL}

The model includes both modification of multiple monomers and a large structural change between the tense (T) 


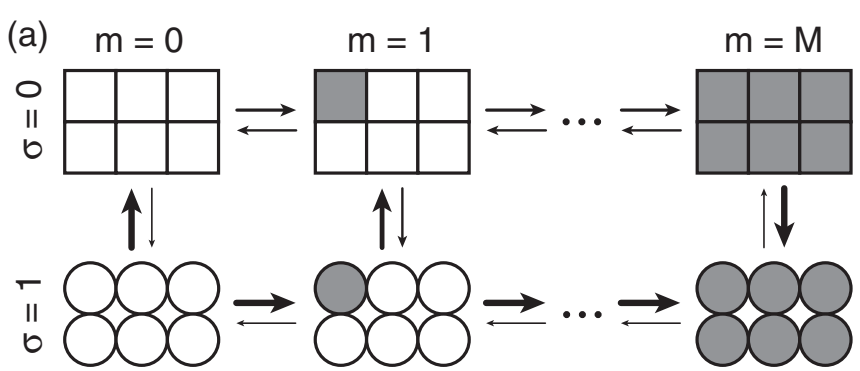

(b)

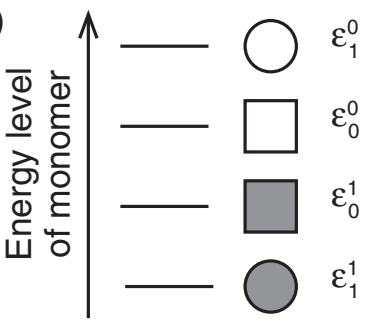

(c)

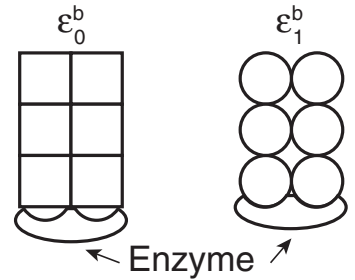

FIG. 1. Schematics of the enzymatic MWC model. (a) All states that a single MWC molecule can take. $\sigma$ represents the T or R state and $m$, the number of modifications. (b) Energy level of each monomer. (c) Binding energies between the enzyme and the MWC molecules with different states. The binding energy depends on a molecular structure $\sigma$.

and relaxed (R) states. We here represent each modification and each structural state as two types of "spins"; that is, each molecule has $M$ modification spins and a single structural spin taking a down or up state, where $M$ is the number of monomers and set as 6 unless otherwise noted. Thus, each molecule has $2^{M+1}$ states. The modification spin $s_{i}$ flips from 0 to 1 when each site is modified, where $i$ is $1 \leqslant i \leqslant M$, and the total number of up spins is denoted as $m$. The structural spin $\sigma$ flips between the $\mathrm{T}(\sigma=0)$ and $\mathrm{R}(\sigma=1)$ state with a structural change. The internal energy of a single molecule is defined as the summation of energies of all modification spins. Thus, the Hamiltonian of the single molecule is given as

$$
\mathscr{H}\left(\sigma,\left\{s_{i}\right\}\right)=\left[m \epsilon_{\sigma}^{1}(h)+(M-m) \epsilon_{\sigma}^{0}\right],
$$

where $\epsilon_{\sigma}^{1}(h)$ and $\epsilon_{\sigma}^{0}$ are the energies of the up and down spins, respectively, of the $\sigma$ state molecule. $\epsilon_{\sigma}^{1}(h)$ is a function of $h$, which is the "chemical field" derived from the concentration of the coenzyme required for the transfer of functional groups. Although the dependence of the MWC model on the ligand concentration has been studied extensively [14,15], here we set $\epsilon_{\sigma}^{1}$ as constant, because we are concerned with enzymatic constraint. $\left\{s_{i}\right\}$ is a set of modification spins. The number of molecules in the present system is fixed at $N$. We set $N$ as 100 unless otherwise noted. Therefore, the partition function is given as

$$
Z=\left[\sum_{\sigma=0,1} \sum_{i}\left(\begin{array}{c}
M \\
i
\end{array}\right) \exp \left\{-\beta\left((M-i) \epsilon_{\sigma}^{0}+i \epsilon_{\sigma}^{1}\right)\right\}\right]^{N} .
$$

We introduce two quantities: the fraction of unmodified monomers $\mathcal{U}$ and the T-state molecule ratio $\mathcal{T}$. These are defined as $1-\Sigma_{j} m_{j} /(N M)$ and $N_{0} / N=1-N_{1} / N$, respectively, where $m_{j}$ is the modification level $m$ of the $j$ th molecule, $N_{0}$ and $N_{1}$ are the numbers of T- and R-state molecules, respectively. Such quantities in the equilibrium state are easily derived from the partition function because there is no interaction term among the molecules in the Hamiltonian. The two types of allosteric effects to accelerate the reaction are formulated as follows.

(i) Energetic effect. If a molecule is modified at many modification sites, such a molecule tends to change from the $\mathrm{T}$ to $\mathrm{R}$ state. The modification sites of $\mathrm{R}$-state proteins are easier to modify than those of T-state proteins. Therefore, the energy of each modification spin has to satisfy the inequality $\epsilon_{1}^{0}>\epsilon_{0}^{0}>\epsilon_{0}^{1}>\epsilon_{1}^{1}$ [Fig. 1(b)]. Here, $\epsilon_{1}^{0}, \epsilon_{0}^{0}, \epsilon_{0}^{1}$, and $\epsilon_{1}^{1}$ are set to $4,3,2$, and 1 , respectively.

By considering the detailed balance condition, the transition probability of each protein state is given as

$$
\begin{aligned}
& \frac{p\left(\sigma,\left\{1, \ldots, s_{M}\right\} \mid \sigma,\left\{0, \ldots, s_{M}\right\}\right)}{p\left(\sigma,\left\{0, \ldots, s_{M}\right\} \mid \sigma,\left\{1, \ldots, s_{M}\right\}\right)}=\frac{\exp \left(-\beta \epsilon_{\sigma}^{1}\right)}{\exp \left(-\beta \epsilon_{\sigma}^{0}\right)}, \\
& \frac{p\left(1,\left\{s_{i}\right\} \mid 0,\left\{s_{i}\right\}\right)}{p\left(0,\left\{s_{i}\right\} \mid 1,\left\{s_{i}\right\}\right)}=\frac{\exp \left[-\beta\left\{m \epsilon_{1}^{1}+(M-m) \epsilon_{1}^{0}\right\}\right]}{\exp \left[-\beta\left\{m \epsilon_{0}^{1}+(M-m) \epsilon_{0}^{0}\right\}\right]},
\end{aligned}
$$

where $p\left(\sigma,\left\{1, \ldots, s_{M}\right\} \mid \sigma,\left\{0, \ldots, s_{M}\right\}\right)$ and $p(\sigma,\{0, \ldots$, $\left.\left.s_{M}\right\} \mid \sigma,\left\{1, \ldots, s_{M}\right\}\right)$ are the transition probabilities for the modification and nonmodification of the first modification spin of a $\sigma$-state molecule, respectively. The same transition probabilities are adopted for the other modification spins. $p\left(1,\left\{s_{i}\right\} \mid 0,\left\{s_{i}\right\}\right)$ and $p\left(0,\left\{s_{i}\right\} \mid 1,\left\{s_{i}\right\}\right)$ are the transition probabilities for the structural change from $\sigma=0$ to $\sigma=1$ and $\sigma=1$ to $\sigma=0$, respectively, when the modification state is $\left\{s_{i}\right\}$.

When $m$ is small, the structure tends to be $\sigma=0$ because $\epsilon_{1}^{0}>\epsilon_{0}^{0}$, whereas the structure tends to be $\sigma=1$ because $\epsilon_{0}^{1}>\epsilon_{1}^{1}$ for large $m$. The transition probabilities from $\sigma=0$ to 1 and $\sigma=1$ to 0 are identical for $m=M / 2$. Here, we assume that the structural change can always occur within the characteristic time of that when the microscopic energy decreases, i.e., we adopt the Metropolis method for structural change.

For the modification, the activation energy is set to be equal to $\epsilon_{1}^{0}$ for all modification reactions for simplicity. Hence, the modification of the R-state molecule has no energy barrier, whereas that of the T-state molecule has an energy barrier.

(ii) Enzymatic (kinetic) effect. Although the enzyme works as a catalyst for modification and does not change the detailed balance condition, competition for the enzyme among molecules introduces a kinetic effect. By assuming that the timescale of enzyme binding is much faster than that of modification and state change, the binding reaction can be eliminated adiabatically. Then, the kinetics of the modification are governed by the product of the binding probability $\left(P_{\sigma}^{\mathrm{b}}\right)$ and the activation probability to go across the energy barrier. Hence, the enzymatic effect is represented by the changes in $P_{0}^{\mathrm{b}}$ and $P_{1}^{\mathrm{b}}$ following the structural change.

To accelerate the reaction, the R-state molecule tends to bind the enzyme more and is modified faster than the $\mathrm{T}$ state, i.e., $P_{1}^{\mathrm{b}}$ is larger than $P_{0}^{\mathrm{b}}$. Thus, the $\mathrm{T}$ - and R-state molecules have different binding energies with the enzyme of $\epsilon_{0}^{\mathrm{b}}$ and $\epsilon_{1}^{\mathrm{b}}$, respectively, where $\epsilon_{0}^{\mathrm{b}}$ is lower than $\epsilon_{1}^{\mathrm{b}}\left(\epsilon_{0}^{\mathrm{b}}=0\right.$ and $\left.\epsilon_{1}^{\mathrm{b}}=10\right)$. Under the assumption that only a single enzyme can bind to 
the molecule, the transition probability for the modification under the detailed balance condition [Eq. (3)] is

$$
\begin{aligned}
p\left(\sigma,\left\{1, \ldots, s_{M}\right\} \mid \sigma,\left\{0, \ldots, s_{M}\right\}\right) & =P_{\sigma}^{\mathrm{b}} \exp \left(-\beta\left\{\epsilon_{1}^{0}-\epsilon_{\sigma}^{0}\right\}\right), \\
p\left(\sigma,\left\{0, \ldots, s_{M}\right\} \mid \sigma,\left\{1, \ldots, s_{M}\right\}\right) & =P_{\sigma}^{\mathrm{b}} \exp \left(-\beta\left\{\epsilon_{1}^{0}-\epsilon_{\sigma}^{1}\right\}\right), \\
P_{\sigma}^{\mathrm{b}}=\frac{\left\langle n_{\sigma}\right\rangle}{N_{\sigma}} & =\frac{\exp (\beta \mu)}{\exp \left(-\beta \epsilon_{\sigma}^{\mathrm{b}}\right)+\exp (\beta \mu)},
\end{aligned}
$$

where $n_{\sigma}$ is the number of enzymes that bind to the $\sigma$-state molecule [16]. The above Eq. (5) for the binding probability corresponds to the Michaelis-Menten equation for multiple substrates $[14,15]$. In particular, if binding energies are sufficiently large and the concentration of free enzyme is asymptotically zero, Eq. (5) is identical to the Michaelis-Menten equation. Figure S1 in the Supplemental Material [16] shows the binding probabilities calculated from Eq. (5).

We set the timescales of the modification flip and state flip as $\tau_{\mathrm{m}}$ and $\tau_{\mathrm{s}}$, respectively, where $\tau_{\mathrm{m}}$ is longer than $\tau_{\mathrm{s}}$ $\left(\tau_{\mathrm{s}}=1.0, \tau_{\mathrm{m}}=10.0\right)$. We set the initial condition such that all molecules are in the $\sigma=0$ and $m=0$ states. If biochemical systems were composed only of one-body reactions, it would be represented by linear dynamics and could be solved analytically $[17,18]$. Our system, however, includes enzymatic reactions and is nonlinear. With competition for the enzyme among $N$ molecules, which respectively take $2^{M+1}$ states, it involves $2^{M+1} N$ possible states. In addition, we here consider the relaxation process to the equilibrium, and then analytic and approximation methods are hard to be applied in general $[18,19]$. Hence, we investigated the relaxation dynamics to the equilibrium state using the Monte Carlo (MC) method to sample across a huge state space.

\section{RESULTS}

First, we calculated the relaxation dynamics of $\mathcal{U}$ and $\mathcal{T}$. Although $\langle\mathcal{U}\rangle_{\text {ens }}$ and $\langle\mathcal{T}\rangle_{\text {ens }}$, where \langle\rangle$_{\text {ens }}$ is the ensemble average, finally relaxed to the equilibrium values $\mathcal{U}_{\text {eq }}$ and $\mathcal{T}_{\text {eq }}$, respectively, their time courses varied depending on the temperature $1 / \beta$ and $\langle n\rangle$, which is the average number of enzymes binding to a substrate (Fig. 2). When the temperature was high, $\langle\mathcal{U}\rangle_{\text {ens }}$ decreased exponentially with time with no plateau. As the temperature decreased, the relaxation slowed down and decreased logarithmically with time. Two plateaus appeared as the temperature decreased further [Fig. 2(a)]. The two plateaus were clearly discernible when $\langle n\rangle$ was reduced below $\langle n\rangle / N=0.95$. The relaxation of $\langle\mathcal{T}\rangle_{\text {ens }}$ also showed a similar dependence on $\langle n\rangle$ and the temperature, as shown in Fig. S2. Such slow dynamics with plateaus have often been observed in glasses [7]. Note that one can derive a deterministic rate equation from the reaction kinetics (5), for investigating just the average temporal evolution of chemical concentrations. The averaged relaxation time courses shown in Fig. 2 perfectly agreed with the deterministic model (see Ref. [16] and Fig. S3), whereas the MC method is required to study the distribution in relaxation paths by fluctuations.

To reveal the mechanism of the anomalous parameter dependence, we analyzed the relaxation-time distribution over the samples. In the region where the relaxation time showed

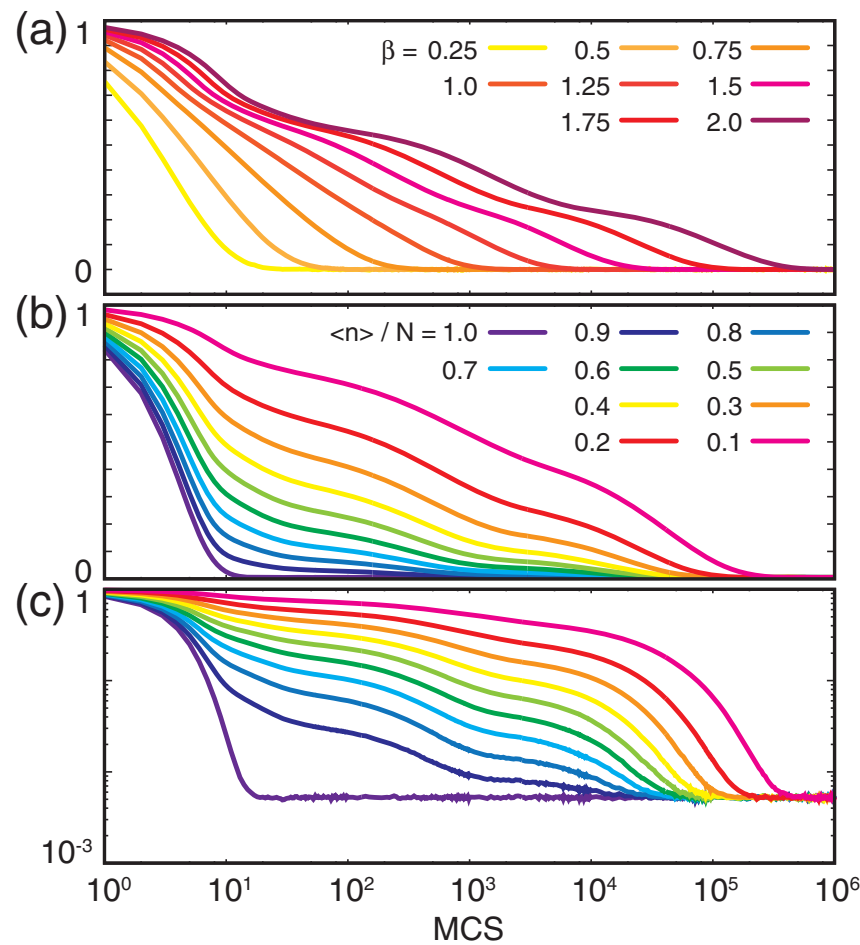

FIG. 2. Time evolution of the average modification in the eMWC against the logarithmic Monte Carlo step. (a) Relaxation of the average modification to the equilibrium at various temperatures. Since $\mathcal{U}_{\text {eq }}$ depends on the temperature, the normalized ratio $\left(\langle\mathcal{U}\rangle_{\text {ens }}-\right.$ $\left.\mathcal{U}_{\text {eq }}\right) /\left(1-\mathcal{U}_{\text {eq }}\right)$ was plotted by setting $\langle n\rangle / N$ at 0.2 . Different color lines indicate time courses with different values of $\beta$. (b) Relaxation of the average modification to the equilibrium for various values of $\langle n\rangle$. The time course of $\langle\mathcal{U}\rangle_{\text {ens }}$ was plotted by setting $\beta$ at 1.75 . The different line colors correspond to different values of $\langle n\rangle / N$. $\left(\mathcal{U}_{\text {eq }}\right.$ is independent of $\langle n\rangle$.) Each line is an ensemble average of 1000 samples. (c) Logarithm of $\langle\mathcal{U}\rangle_{\text {ens }}$ plotted for (b).

an anomalous parameter dependence, the relaxation-time distribution changed from unimodal to multimodal (Fig. 3). The multiple peaks that emerged are named the first, second, and third peaks in ascending order of relaxation time.

When $\langle n\rangle$ was varied at a fixed temperature, the positions of the peaks changed in proportion to $\langle n\rangle^{-1}$ [Fig. 3(a)]. Thus, we studied the distribution of the relaxation time normalized by $\langle n\rangle^{-1}$. When $\langle n\rangle / N$ was close to 1 , two peaks were observed. As $\langle n\rangle$ decreased, the first peak disappeared and was replaced by the third one. Finally, the second peak disappeared completely at $\langle n\rangle / N=0.4$. This change in the distribution is similar to the first-order phase transition in equilibrium thermodynamics. Indeed, as $M$ was increased, the divergence of the relaxation time against $\langle n\rangle / N \rightarrow 1$ was steeper [Fig. 3(d)] and its variance increased (Fig. S4). This suggests that in the limit of $M \rightarrow \infty$ and $N \rightarrow \infty$, the change in the relaxation time is similar to the phase transition in the context of equilibrium thermodynamics. Actually, as $N$ increased, the divergence of the relaxation time was steeper even in the case of $M=6$ (Fig. S5). It should be recalled that the same equilibrium state was reached over all samples independent of the relaxation courses, and the transition here 

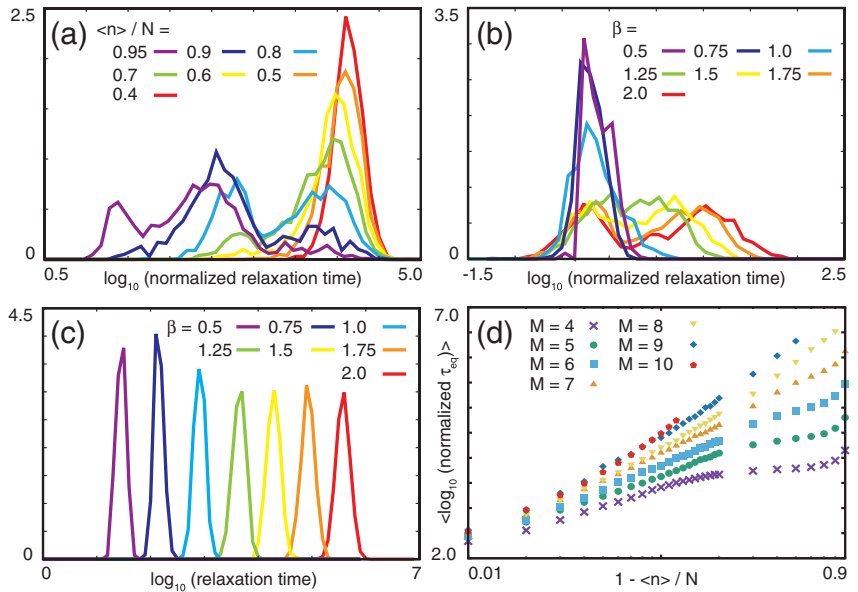

FIG. 3. Distributions of the relaxation time and the variance of the logarithmic relaxation time. Probability distribution of the logarithmic normalized relaxation time at various $\langle n\rangle$ values with fixed temperature at (a) $\beta=1.75$, and at various temperatures with $\langle n\rangle / N$ fixed at (b) 0.8 and (c) 0.2 , respectively. The logarithmic relaxation time was calculated as the base-10 logarithm of $\tau_{\text {eq }}$. Plots are rescaled by $\tau_{\text {eq }}\langle n\rangle^{-1}$ for (a) and rescaled by $\tau_{\text {eq }} e^{-4 \beta}$ for (b). Different color lines indicate the probability distributions under different parameters. (d) Averaged normalized relaxation time for different number of modification sites $M$ indicated by different symbols. The plot is rescaled in the similar way as in (a).

is with regard to the relaxation trajectories rather than the quantities in thermodynamic equilibrium.

The temperature dependence of the relaxation-time distribution when $\langle n\rangle / N$ was fixed close to 1 differed from its $\langle n\rangle$ dependence [Fig. 3(b)]. In this case, the position of the first peak changed in proportion to $\exp (4 \beta)$, as explained later. At high temperatures, the relaxation-time distribution was unimodal. This distribution broadened with decreasing temperature at around $\beta=1.0$, where it became bimodal, and the distance between these two peaks increased with decreasing temperature. This change in the relaxation-time distribution is similar to the second-order phase transition in equilibrium, whereas when $\langle n\rangle / N$ is small, the relaxation-time distribution shows not a transition but a crossover [Fig. 3(c)]. Indeed, the variance of the logarithmic relaxation time is large at around $\langle n\rangle / N=1$ [Fig. S6(a)].

This transition is caused by kinetic constraint from the competition for the enzyme among R- and T-state molecules. As the relaxation progresses and the number of $\mathrm{R}$-state molecules reaches almost the same level as that of the enzyme, the R-state molecules monopolize the enzyme due to the positive allosteric effect (Fig. S1). Then, further progress in the modification reactions of the T-state molecules is suppressed (Fig. 4). Then, the transition from the $\mathrm{T}$ to the $\mathrm{R}$ state is rate limiting with three possible steps, $m=0,1$, and 2, having the energy barriers 6,4 , and 2 , respectively. Thus, the temperature dependence of the transition rate follows $\exp (6 \beta)(m=0)$, $\exp (4 \beta)(m=1)$, and $\exp (2 \beta)(m=2)$.

When the number of molecules is finite, the rate-limiting step depends on the distribution of the phosphorylation level of the T-state molecules at the start of the kinetically constrained condition. As long as the $(m=0, \sigma=0)$ molecule

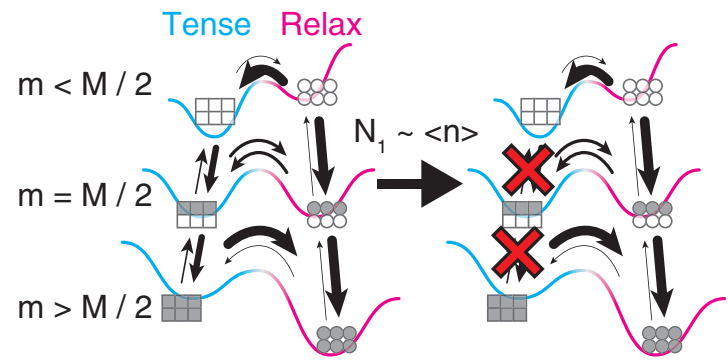

FIG. 4. Schematic showing the energy landscape of the eKCM. Left: If the number of R-state molecules is lower than that of the enzyme, the modification reaction of T-state molecules (up and down arrows) can occur and progress until half of the modification sites are modified. Then, when more than half of the sites are modified, such molecules tend to be in the R state (lower right arrow). Right: When the number of R-state molecules exceeds that of the enzyme through modification, the R-state molecules monopolize the enzyme, and the modification reaction of the T-state molecules is kinetically inhibited (red crosses). Therefore, the structural changes from the $\mathrm{T}$ to $\mathrm{R}$ state in the less modified molecules (upper right arrow) are rate limiting.

exists, the transition from ( $m=0, \sigma=0)$ to $(m=0, \sigma=1)$ is rate limiting, whereas if it is not included, its transition from $(m=1, \sigma=0)$ to $(m=1, \sigma=1)$ is rate limiting as long as the $m=1$ molecule exists, and so forth. These three rate-limiting steps, therefore, correspond to the third, second, and first peaks in Fig. 3(a), respectively, and the three plateaus of relaxation (Fig. 2). Indeed, only the samples in the peak with the longest relaxation time and having three plateaus had molecules in the $m=0$ state (Fig. S7). This suggests that the fluctuation in the distribution of molecular states at the beginning of relaxation affects the entire process in the trajectory.

Reflecting such a transition in relaxation processes, the average relaxation time shows an anomalous dependence on $1 / \beta$ (Figs. 5 and S8). When $\langle n\rangle$ was fixed and the temperature was varied, the relaxation time did not follow the standard Arrhenius form [Fig. 5(a)], similar to the glass. Because the energy barrier for the modification of the T-state molecule was set to unity while the modification of the R-state molecule was temperature independent, the relaxation time would normally be expected to be proportional to $\exp (\beta)$. Indeed, at high
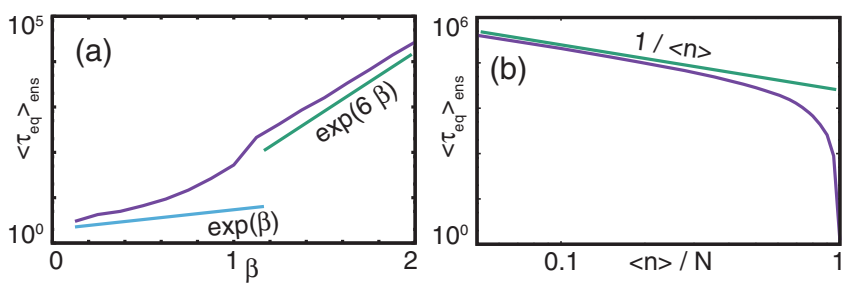

FIG. 5. Relaxation times at various temperatures and $\langle n\rangle$ values. (a) Dependence of the relaxation time on the temperature. The relaxation time is defined as the ensemble average of $\tau_{\text {eq }}$ when $\mathcal{T}$ falls below the analytically calculated value $\mathcal{T}_{\text {eq }}$ because $\mathcal{T}$ approaches equilibrium from above. The blue and green lines indicate lines proportional to $\exp (\beta)$ and $\exp (6 \beta)$, respectively. $\langle n\rangle / N$ was set at 0.8. (b) Dependence of the relaxation time on $\langle n\rangle / N$. The green line indicates a line proportional to $\langle n\rangle^{-1}$. $\beta$ was set at 1.75 . 
temperatures, the relaxation time approximately followed $\exp (\beta)$. However, as the temperature decreased, the rate of increase in the relaxation time against the temperature was enhanced and reached its maximum at around $\beta=1$. The relaxation time showed a bending point at around $\beta \sim 1$, and for low temperatures, it approximately obeyed $\exp (6 \beta)$. This dependence suggests that the transition from the $(m=0, \sigma=$ $0)$ to $(m=0, \sigma=1)$ is rate limiting, whose activation energy is six times the energy barrier for each modification (Fig. 1).

The relaxation time also shows an anomalous dependence on $\langle n\rangle$ around $\langle n\rangle / N=1$ [Fig. 5(b)], where the transition of distribution of relaxation time is observed. In the ordinal chemical reaction, the relaxation time changes in proportion to the number of the complex $\langle n\rangle$, in accordance with the binding probability. Indeed, for lower $\langle n\rangle$, the relaxation time was proportional to the inverse of $\langle n\rangle$. However, it was further prolonged beyond $\langle n\rangle^{-1}$ as $\langle n\rangle / N$ decreased to approach 1 .

\section{DISCUSSION}

Here, we propose a statistical physics model to adopt the kinetic constraint concept to biochemical systems. In our model, the kinetic constraint is autonomously imposed by competition for the enzyme among T- and R-state molecules, resulting in the glassy relaxation. In consistency with the standard $\mathrm{KCM}$, the present kinetic constraint is generated and controlled by the enzyme abundance, which works as a parameter for the phase transition; therefore, it is termed as eKCM.

The relaxation of modification of the T-state molecules is frozen by the competition for the enzyme, whereas the $\mathrm{R}$-state molecules are partially equilibrated. This is similar to the dynamical heterogeneity, important in glass theory [20]. The number of frozen molecules depends on the history and differs for each replicate (Figs. S9 and S10). The variance in the modification level approaches $M$ through the relaxation (Fig. S9). This reflects the MWC-type allostery, where all the $M$ monomers flip their molecular structure cooperatively.

The eKCM exhibited the transition in the relaxation paths to equilibrium, depending on the temperature and the enzyme concentration. Following the transition of the trajectories, slow relaxation and plateaus appeared, as in the glass transition. Most of the KCMs studied thus far, however, do not show the transition of paths with a change in temperature [8], and needless to say, there is no external parameter corresponding to the enzyme abundance. In the eKCM, the strength of the cooperativity in kinetics depends on the enzyme abundance and temperature. When the amount of enzyme is greater than that of the substrate, there is no competition, and glassy behavior does not appear. The competition for a limited amount of the enzyme introduces interactions among molecules, resulting in the transition to a state with heterogeneity in the relaxation paths.

Our study also demonstrated that microscopic fluctuation in molecular states can be amplified to a large variation in the relaxation time. Although the noise in chemical concentrations has recently attracted much attention from many physicists and biologists [21], there has been little study on the fluctuations in the relaxation paths in biology. They can be easily observed experimentally by using a biochemical reaction in a liposome or emulsion, as larger fluctuations are expected for a system with a small number of molecules.

In recent decades, the slow relaxation process has also attracted much interest among biophysicists. In bacterial chemotaxis, chemoreceptors, which are often described by an MWC-type model [22,23], are known to form clusters with each other and show a slow logarithmic change in their structure with time in response to the addition and removal of a ligand [24]. Other example is the phosphorylation of PER 2 by a kinase, $\mathrm{CKI} \epsilon / \delta$, which determines the period of the mammalian circadian clock [25]. Interestingly, $\mathrm{CKI} \epsilon / \delta$ tends to rebind its own catalytic products, and mutant mice with a lower rebinding activity showed a shorter period of circadian rhythm [26], which corresponds to our results. We expect that the experiments with the above systems will demonstrate the slow relaxation of modifications as well as a large variance of relaxation time, and deepen our understanding of the relationship between the regulation of biological timescales and glass theory in physics.

\section{ACKNOWLEDGMENTS}

We thank Atsushi Ikeda for a critical reading of the manuscript and Yasushi Okada and Tom Shimizu for helpful discussions. This work was partially supported by Grantsin-Aid for Scientific Research, KAKENHI, Grants No. 17H05758 and No. 15H05746, and a Grant-in-Aid for Scientific Research on Innovative Areas from the Ministry of Education, Culture, Sports, Science and Technology (MEXT) of Japan, Grant No. 17H06386.
[1] R. Phillips, J. Kondev, and J. Theriot, Physical Biology of the Cell (Garland Science/Taylor \& Francis, New York, 2008).

[2] A. Mylona, F. X. Theillet, C. Foster, T. M. Cheng, F. Miralles, P. A. Bates, P. Selenko, and R. Treisman, Opposing effects of Elk-1 multisite phosphorylation shape its response to ERK activation, Science 354, 233 (2016).

[3] C. Salazar and T. Höfer, Multisite protein phosphorylationFrom molecular mechanisms to kinetic models, FEBS J. 276, 3177 (2009).

[4] T. S. Hatakeyama and K. Kaneko, Generic temperature compensation of biological clocks by autonomous regulation of catalyst concentration, Proc. Natl. Acad. Sci. USA 109, 8109 (2012).

[5] T. S. Hatakeyama and K. Kaneko, Kinetic memory based on the enzyme-limited competition, PLoS Comput. Biol. 10, e1003784 (2014).

[6] M. Zhou, J. K. Kim, G. W. L. Eng, D. B. Forger, and D. M. Virshup, A period2 phosphoswitch regulates and temperature compensates circadian period, Mol. Cell 60, 77 (2015).

[7] P. G. Debenedetti and F. H. Stillinger, Supercooled liquids and the glass transition, Nature (London) 410, 259 (2001). 
[8] F. Ritort and P. Sollich, Glassy dynamics of kinetically constrained models, Adv. Phys. 52, 219 (2003).

[9] J. Monod, J. Wyman, and J.-P. Changeux, On the nature of allosteric transitions: A plausible model, J. Mol. Biol. 12, 88 (1965).

[10] J. Wyman and S. J. Gill, Binding and Linkage: Functional Chemistry of Biological Macromolecules (University Science Books, Mill Valley, CA, 1990).

[11] E. Di Cera, Thermodynamic Theory of Site-Specific Binding Processes in Biological Macromolecules (Cambridge University Press, Cambridge, UK, 1995).

[12] A. Y. Ben-Naim, Statistical Thermodynamics for Chemists and Biochemists (Plenum, New York, 1998).

[13] S. Marzen, H. G. Garcia, and R. Phillips, Statistical mechanics of Monod-Wyman-Changeux (MWC) models, J. Mol. Biol. 425, 1433 (2013).

[14] I. H. Segel, Enzyme Kinetics: Behavior and Analysis of Rapid Equilibrium and Steady-State Enzyme Systems (Wiley, New York, 1993).

[15] A. Cornish-Bowden, Fundamentals of Enzyme Kinetics (WileyBlackwell, Weinheim, 2013).

[16] See Supplemental Material at http://link.aps.org/supplemental/ 10.1103/PhysRevResearch.2.012005 for the derivation.

[17] H. Qian, Open-system nonequilibrium steady state: Statistical thermodynamics, fluctuations, and chemical oscillations, J. Phys. Chem. B 110, 15063 (2006).
[18] D. Schnoerr, G. Sanguinetti, and R. Grima, Approximation and inference methods for stochastic biochemical kinetics-A tutorial review, J. Phys. A: Math. Theor. 50, 093001 (2017).

[19] N. G. van Kampen, Stochastic Processes in Physics and Chemistry (Elsevier, Amsterdam, 1992).

[20] Dynamical Heterogeneities in Glasses, Colloids, and Granular Media, edited by L. Berthier, G. Biroli, J.-P. Bouchaud, L. Cipelletti, and W. van Saarloos (Oxford University Press, New York, 2011).

[21] M. B. Elowitz, A. J. Levine, E. D. Siggia, and P. S. Swain, Stochastic gene expression in a single cell, Science 297, 1183 (2002).

[22] S. Asakura and H. Honda, Two-state model for bacterial chemoreceptor proteins: The role of multiple methylation, J. Mol. Biol. 176, 349 (1984).

[23] G. Lan and Y. Tu, Information processing in bacteria: Memory, computation, and statistical physics: A key issues review, Rep. Prog. Phys. 79, 052601 (2016).

[24] V. Frank and A. Vaknin, Prolonged stimuli alter the bacterial chemosensory clusters, Mol. Microbiol. 88, 634 (2013).

[25] Y. Isojima et al., CKI $\epsilon / \delta$-dependent phosphorylation is a temperature-insensitive, period-determining process in the mammalian circadian clock, Proc. Natl. Acad. Sci. USA 106, 15744 (2009).

[26] Y. Shinohara et al., Temperature-sensitive substrate and product binding underlie temperature-compensated phosphorylation in the clock, Mol. Cell 67, 783 (2017). 